\title{
Role of the Transcriptional Repressor BCL6 in Allergic Response and Inflammation
}

\author{
Masafumi Arima,* Takeshi Fukuda, $\dagger$ and Takeshi Tokuhisa*
}

\begin{abstract}
Various molecules participate in different phases of allergic reactions. This means that many genes are encoding molecules related to allergic reactions, such as cytokines, chemokines, and their receptors as effector molecules. The transcriptional repressor BCL6 has emerged as a multifunctional regulator of lymphocyte differentiation and immune responses. BCL6-deficient $\left(\right.$ BCL6 $^{-1-}$ ) mice display $\mathrm{T}$ helper type 2 (Th2)-type inflammation, which is caused by abnormality of both lymphoid cells and nonlymphoid cells. Thus, BCL6 apparently contributes to negative regulation of various central molecules such as cytokines, in particular Th2 cytokines, CC chemokines, and immunoglobulin E in allergic diseases. Therefore, BCL6 may be a molecular target for Th2-type allergic diseases.
\end{abstract}

Key Words: allergy, BCL6, cytokine, chemokine, $\lg$, Th2, transcriptional repressor

(WAO Journal 2008;115-122)

A llergic diseases are characterized by elevated serum immunoglobulin E ( IgE) levels and hypersensitivity to normally innocuous antigens (allergens). A particular direct allergen first encounters antigen-processing cells such as dendritic cells (DCs) or macrophages. The allergen is captured by the antigen-processing cells, processed, and presented to $\mathrm{CD}^{+} \mathrm{T}$ cells. $\mathrm{CD} 4^{+} \mathrm{T}$ cells are polarized into $\mathrm{T}$ helper type 1 (Th1) cells, producing interferon- $\gamma$ and interleukin 2 (IL-2), and Th2 cells, producing IL-4, IL-5, IL-6, IL-10, and IL-13. Interleukin 4 and IL-13 stimulate immunoglobulin class switching, leading to the production of $\operatorname{IgE}$, which binds to its high-affinity receptor (FceRI) on the surface of mast cells or basophils. The association of captured allergens with IgE bound to FceRI on the cell surface activates signal transduction in the cells and rapidly leads to the release of inflammatory cytokines and chemical mediators, such as histamine and leukotrienes. The Th2-type cytokines also trigger the production of chemokines in tissue fibroblasts or epithelial cells, promoting the infiltration of inflammatory cells into sites exposed to allergens.

\section{Received for publication March 28, 2008; accepted April 25, 2008.}

From the *Department of Developmental Genetics, Chiba University Graduate School of Medicine, Chiba; and $\dagger$ Department of Pulmonary Medicine and Clinical Immunology, Dokkyo University School of Medicine, Dokkyo, Japan.

The authors declare no conflict of interest.

Reprints: Takeshi Fukuda, Department of Pulmonary Medicine and Clinical Immunology, Dokkyo University School of Medicine, 880 Mibu-machi Shimotsuga-gun, Tochigi 321-0293, Japan. E-mail: t-fukuda@ dokkyomed.ac.jp

Copyright (c) 2008 by World Allergy Organization
Various allergy-related molecules are basically controlled by transcriptional regulators, critical molecules believed to govern the pathogenesis of allergic diseases by regulating cytokine production, mediator synthesis, and $\operatorname{IgE}$ production at gene expression levels. Recent genetic studies have demonstrated that transcriptional factors and regulators are involved in the development of allergic diseases. The molecular mechanisms of allergic diseases, in particular bronchial asthma, are gradually becoming clearer. However, nearly all previous studies of transcriptional factors have focused on active regulators, including GATA binding protein 3 (GATA3), signal transducer and activator of transcription 6 (STAT6), c-Maf, NF-AT, NF-кB, and c-fos. The functional roles of transcriptional repressors in allergic diseases remain poorly understood. Because transcriptional repressors may have an important role in tuning physiologically optimal transcription, functional failure of tightly controlled constitutive mechanisms regulated by a given repressor may lead to the development of allergic diseases.

A transcriptional repressor gene, $B C L 6$, has emerged as a multifunctional regulator of lymphocyte differentiation and immune responses. ${ }^{1,2}$ BCL6 mutant mice display two prominent phenotypes: failure to form germinal centers during $\mathrm{T}$ cell-dependent immune responses and fatal eosinophilic inflammatory diseases characterized by the presence of Th2 cells and mast cells. ${ }^{3-5}$ Although the molecular mechanisms underlying these phenotypes are largely unknown, studies in BCL6-deficient mice have suggested that BCL6 functions to prevent the development or attenuate the pathogenesis of allergic diseases. In this article, we review the functions of BCL6 in allergic diseases by focusing on recent data from our laboratories and from other groups.

\section{STRUCTURE AND BASIC FUNCTIONS OF BCL6}

The human proto-oncogene $B C L 6$ was first identified in studies of chromosomal breakpoints involving 3q27 in diffuse large B-cell lymphomas. ${ }^{6-8}$ BCL6 is expressed at low levels in a wide variety of tissues. It is expressed abundantly only in germinal center B cells, cortical thymocytes, and parafollicular T cells within secondary lymphoid tissues. ${ }^{9}$ High BCL6 levels are also present in cells of monocytic lineage. ${ }^{10}$ The BCL6 gene encodes a 92- to $98-\mathrm{kd}$ nuclear phosphoprotein that contains the $\mathrm{BTB} / \mathrm{POZ}$ domain in the $\mathrm{NH}_{2}$-terminal region and Krüppel-type zinc finger motifs in the $\mathrm{COOH}$-terminal region (Fig. 1). Because the $\mathrm{NH}_{2}$-terminal half of BCL6 can bind to silencing mediator of retinoid and thyroid receptor protein (SMRT) and recruit the SMRT/histone deacetylase complex to silencer regions of target genes to repress the expression of these genes, BCL6 can function as a sequence-specific 
Bcl6 Transcriptional Repressor Activity

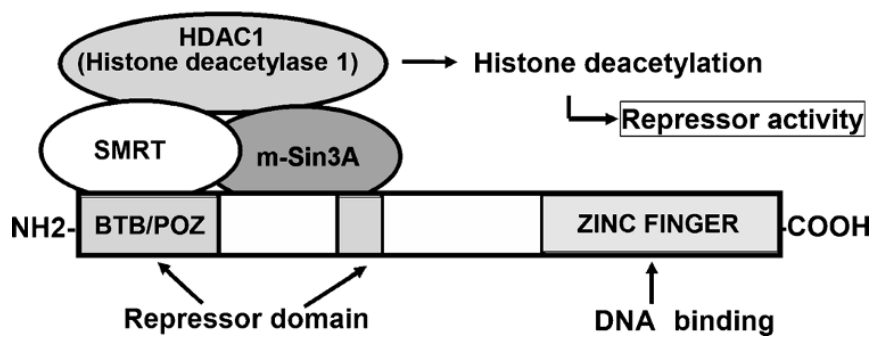

FIGURE 1. Structure of BCL6. The BCL6 gene encodes a 92- to 98-kd nuclear phosphoprotein that contains the BTB/POZ domain in the $\mathrm{NH}_{2}$-terminal region and Krüppel-type zinc finger motifs in the $\mathrm{COOH}$-terminal region. The $\mathrm{NH}_{2}$-terminal half of BCL6 can bind to SMRT and recruit the SMRT/histone deacetylase complex to silencer regions of target genes to repress the expression of these genes. Because the BCL- 6 zinc-fingers bind DNA in a sequence-specific manner, $B C L 6$ can function as a sequence-specific transcriptional repressor.

transcriptional repressor. The BCL6 zinc-fingers bind to DNA in a sequence-specific manner, and a consensus BCL6 DNAbinding site has been identified. ${ }^{11,12}$ The BCL6 consensus binding site resembles the GAS motif recognized by the STAT family of transcription factors, raising speculation that BCL6 may bind competitively to some STAT-binding sites to repress expression of STAT-dependent genes. ${ }^{3,13,14}$ Target genes of BCL6 have been identified. Available evidence indicates that BCL6 is a multipotential molecule because its target genes are related to various molecules, including cytokines, chemokines, cell cycle regulators, DNA damage-related proteins, apoptosisrelated proteins, and transcriptional factors (Fig. 2). ${ }^{3,15-31}$

When overexpressed, BCL6 can inhibit cell growth and even induce programmed cell death in nonlymphoid cells such as fibroblasts. ${ }^{32,33}$ This finding indicates that BCL6 is toxic to cells that do not normally express the protein at high levels. Although this fact suggests an important role of BCL6 in normal cell growth, its relevance to normal cell physiology is unclear. We have recently demonstrated that BCL6 is essential for the generation of high-affinity memory B cells in germinal centers $^{4,34}$ and that BCL6 controls the generation and maintenance of memory $\mathrm{CD}^{+} \mathrm{T}$ cells $\mathrm{s}^{35,36}$ and memory CD4 T cells. ${ }^{37}$ Our findings suggest that Bcl6 in T cells plays a role in protecting memory precursor $\mathrm{T}$ cells from apoptosis and may be involved in the survival of long-term memory $\mathrm{T}$ cells.

\section{FUNCTIONS OF BCL6 IN B CELLS}

Although BCL6 messenger RNA (mRNA) can be detected in many tissues, ${ }^{38}$ its protein expression is limited mainly to lymphocytes. ${ }^{38-40}$ BCL6 expression is strongly induced in splenic B cells stimulated with IL-4 or IL-21. ${ }^{41}$ Although IL-2 and IL-7 mediate their biological effects through a common cytokine receptor gamma chain, which is shared with receptors for IL-4 and IL-21, stimulation with these cytokines did not induce high BCL6 expression in splenic B cells. Kinetic analysis demonstrated that BCL6 expression was transiently induced in B cells 30 minutes after stimulation with IL-4 or IL-21. BCL6 expression was further induced in these activated B cells within 6 hours after stimulation with IL-21. In contrast, the recurrence of IL-4-induced BCL6 expression was delayed, occurring 24 hours after stimulation. These findings suggested that IL-4 and IL-21 might be required for high BCL6 expression in B cells at different stages. Stimulation of splenic B cells with IL-4 or IL-21 induced high BCL6 expression with activation of STAT6 or STAT3, respectively, and binding of these STATs to the STAT/BCL6-binding sites in the BCL6 promoter was detected in germinal center B cells in mice. Interleukin 4 and IL-21 may thus have physiological roles in promoting high $B C L 6$ expression in germinal center B cells, and BCL6 induced by STATs activated with IL-4 and/or IL-21 might autoregulate its promoter by negative feedback inhibition.

BCL6 can repress the IL-4-dependent induction of immunoglobulin germ line transcripts by STAT6. Consistent with the role of BCL6 in modulating transcription from the germ line promoter, BCL6 $6^{-1-}$ mice display an increased ability to class switch to IgE in response to IL-4 in vitro. ${ }^{30,31}$ These animals also exhibit a multiorgan inflammatory disease characterized by the presence of large numbers of $\operatorname{IgE}^{+} \mathrm{B}$ cells. The apparent regulation of IgE production is abolished in STAT6 ${ }^{-1-}$ BCL6 $^{-1-}$ mice, indicating that BCL6-dependent regulation of immunoglobulin class switching is STAT6 dependent. Thus, BCL6 can modulate the transcription of selective STAT6-dependent IL-4 responses, including IgE class switching in B cells. Furthermore, we recently simultaneously stimulated activated B cells with IL-4 and IL-21 and found that expression of $\mathrm{C} \gamma 1$ germ line transcript (for $\mathrm{IgG} 1$ class switching) in BCL6 wild type (Wt) and BCL6 ${ }^{-1-}$ B cells was enhanced by IL-21 stimulation, indicating that IL-21 is an enhancer of $\mathrm{C} \gamma 1$ expression induced by IL-4. ${ }^{42}$ The amount of $\mathrm{C} \gamma 1$ germ line transcript in the BCL6 ${ }^{-/-} \mathrm{B}$ cells was greater than that in the BCL6-Wt B cells. Conversely, IL-21 stimulation suppressed $\mathrm{C} \varepsilon$ (for $\operatorname{IgE}$ class switching) expression in the BCL6-Wt B cells. In contrast, such suppression was not observed in the BCL6 ${ }^{-/-} \mathrm{B}$ cells, suggesting that the IL-21-mediated suppression of $\mathrm{C} \varepsilon$ expression is caused by BCL6. Thus, BCL6 controls $\mathrm{C} \gamma 1$ and $\mathrm{C} \varepsilon$ expression and stabilizes class switching to IgG1 in activated B cells simultaneously stimulated with IL-4 and IL-21.

CD23, also known as Fc epsilon RII or FceRII, is the low-affinity receptor for IgE. This antibody isotype is involved in allergy and resistance to parasites and has an important role in the regulation of IgE levels. Unlike many other antibody 


\begin{tabular}{|c|c|c|c|}
\hline Target gene & category & Regulation by BCL6 & Ref\# \\
\hline$C D 23$ & Fc $\varepsilon$ RII & repressor & 3 \\
\hline$I L-5$ & Th2 cytokine & repressor & 15 \\
\hline$I L-6$ & cytokine & repressor & 18 \\
\hline$I L-18$ & cytokine & repressor & 17 \\
\hline$M C P-1$ & chemokine & repressor & 19 \\
\hline$M C P-3$ & chemokine & repressor & 19 \\
\hline$M R P-1$ & chemokine & repressor & 19 \\
\hline IP10 & chemokine & repressor & 20 \\
\hline$M I P-1 \alpha$ & chemokine & repressor & 20 \\
\hline Blimp-1 & $\begin{array}{l}\text { transcriptional repressor } \\
(\mathrm{B}-\text { cell differentiation) }\end{array}$ & repressor & 20 \\
\hline$N F-\kappa B$ & Major transcriptional factor & repressor & 29 \\
\hline GATA3 & $\begin{array}{l}\text { transcriptional factor } \\
\text { (Th2 differentiation) }\end{array}$ & posttranscriptional modification & 16 \\
\hline$C D 80$ & costimulatory receptor & repressor & 21 \\
\hline Cyclin D2 & cell cycle regulator & repressor & 20 \\
\hline$p 53$ & DNA damage-related protein & repressor & 23 \\
\hline$A T R$ & DNA damage-related protein & repressor & 25 \\
\hline$p 21$ & DNA damage-related protein & corepressor & 22 \\
\hline$p 19(A R F)$ & senescence & repressor & 24 \\
\hline$P D C D 2$ & apoptosis & repressor & 26,27 \\
\hline$B C L-x L$ & antiapoptosis & repressor & 28 \\
\hline$I \varepsilon$ & IgE class switch & repressor & 30,31 \\
\hline
\end{tabular}

FIGURE 2. Target genes of BCL6. Previous studies indicate that BCL6 acts as a multipotential molecule because its target genes are for various molecules, including cytokines, chemokines, cell cycle regulators, DNA damage-related proteins, apoptosis-related proteins, and transcriptional factors. ATR indicates ataxia telangiectasia and Rad3 related; IP10, interferon-inducible protein 10; MIP-1 $\alpha$, macrophage inflammatory protein $1 \alpha$; MRP-1, macrophage inflammatory protein-related protein 1; PDCD2, programmed cell death 2 .

receptors, CD23 is a C-type lectin. It is found on not only mature B cells, but also activated macrophages, eosinophils, follicular DCs, and platelets. BCL6 also binds to the STAT6 DNA-binding sequence in the $\mathrm{CD} 23 \mathrm{~b}$ promoter to repress IL-4-induced activation of CD23 expression. ${ }^{3}$ Therefore, BCL6 may be involved in the control of IgE production and act as a negative regulator on one of its ligands.

\section{ROLES OF BCL6 IN T-CELL FUNCTION}

$\mathrm{BCL}^{-1-}$ mice show inflammatory responses in multiple organs, especially the heart and lung, characterized by eosinophil infiltration in young adults., $3,41,43$ BCL6 is considered to regulate Th2-cell differentiation and/or Th2 cytokine production after differentiation. Several groups have investigated the roles of Th2-cell function.
Similar to the key regulators T-bet for Th1 and GATA3 for Th2, human BCL6 was differentially and reciprocally regulated during early Th1- and Th2-cell polarization. At the protein level, the expression of BCL6 was barely detectable in T-helper precursor cells. At the mRNA level, BCL6 is preferentially expressed in cells induced to polarize in the Th1 direction. BCL6 is expressed within 6 hours, and are maintained for at least 48 hours. In contrast, a low level of BCL6 expression is seen in cells induced to polarize in the Th2 direction, apparently because IL-4 down-regulates the human BCL6 gene at an early stage. ${ }^{4,45}$ The differences in BCL6 between Th1- and Th2-polarization decreased or vanished 48 hours after each skewing.

Similar to human BCL6 during the early stage of differentiation into helper T cells, murine BCL6 protein is rapidly induced in naive $\mathrm{T}$ cells induced to polarize in the Th1 
direction, whereas it is induced at a much lower level in T cells skewed toward the Th2 phenotype rather than the Th1 phenotype. The induction of early BCL6 gene expression was transient. Interestingly, Th2-skewed cells showed reinduction of BCL6 at a moderately higher level than Th1-skewed cells did within 7 days during the differentiation of each cell type. ${ }^{46}$ We also found that BCL6 protein was transiently degraded in polarized Th2 cells by T-cell receptor-mediated restimulation, followed by another transient increase in BCL6 protein after 24 hours. In addition, BCL6 gene expression was inhibited by anti-IL- 4 antibodies in polarized Th2 cells after restimulation. Thus, IL-4 may have a bipotential effect and attenuate as well as augment BCL6 production. The machinery for down-regulation of BCL6 remains unclear, whereas its upregulation might involve STAT6-mediated activation of BCL6 promoter in B cells. Furthermore, autoregulation of BCL6 promoter by negative feedback inhibition may occur subsequently, as seen in B cells. Therefore, IL-4 may participate physiologically in the differentiation and reactivation of Th2 cells by tuning the expression of BCL6, which functions on target gene by competing with STATs.

Differentiation of the Th2 pathway has a critical role in determining the outcomes of immune responses. Mediators of Th2 differentiation have been partly elucidated and include a number of cytokines and related signaling molecules. Antigen triggering of naive T cells in the presence of IL- 4 generates a Th2 response. Interleukin 4 -induced gene activation in the Th2 response is mediated by STAT6. ${ }^{47}$ Dent et al ${ }^{48}$ have shown that $\mathrm{IL}_{-} 4^{-1-} \mathrm{BCL}^{-/-}$and STAT6 ${ }^{-1-} \mathrm{BCL6}^{-1-}$ double-mutant mice have the same Th2-type inflammation of the heart and lungs as that characteristically occurring in BCL6 $^{-1-}$ mice. Furthermore, the Th2 cytokine response that develops in STAT6 ${ }^{-1-}$ BCL6 $^{-1-}$ and IL-4 $4^{-1-}$ BCL6 $^{-1-}$ mice is similar to that in BCL6 $6^{-/-}$mice after immunization with a conventional antigen in adjuvant. In contrast to these in vivo findings, STAT6 was required for the in vitro differentiation of BCL6 ${ }^{-/-}$T cells into Th2 cells. BCL6 can bind to similar DNA-binding motifs as STAT transcription factors, suggesting that BCL6 regulates Th2 responses in vivo through a pathway(s) unrelated to IL-4 and STAT6. Kusam et $\mathrm{al}^{16}$ have investigated the mechanism of BCL6 regulation during Th2cell differentiation in vitro and have found that IL-6 signaling can promote dramatically increased levels of Th2 differentiation in BCL6 $6^{-1-}$ CD4 T cells, as compared with BCL6-Wt CD4 T cells. Interleukin 6 could induce Th2 cytokine expression in STAT6 $^{-1-}$ BCL6 $^{-1-}$ cells, but not in BCL6 ${ }^{-1-}$ cells. They also studied whether GATA3 levels are upregulated in STAT6 ${ }^{-1-} \mathrm{BCL6}^{-1-} \mathrm{CD} 4 \mathrm{~T}$ cells as compared with STAT6 ${ }^{-1-}$ CD4 T cells. Retrovirus-mediated expression of BCL6 in both STAT6 ${ }^{-1-}$ BCL6 $^{-1-}$ and BCL6-Wt T cells led to a significant decrease in GATA3 protein levels, thought to be regulated at a post-transcriptional level. Thus, overproduction of Th2 cytokines by BCL6 ${ }^{-1-}$ T cells cannot be explained by loss of competitive inhibition of STAT6 activity, and the regulation of GATA 3 protein levels by BCL6 is probably a key mechanism by which BCL6 regulates STAT6-independent Th2 cytokine expression and Th2 differentiation. The factors that control this alternative pathway have yet to be identified.
In vitro Th2 cells that have differentiated from BCL6-Wt and BCL6 ${ }^{-1-}$ naive T cells produce cytokines other than IL-5 at similar levels after activation, respectively. Interleukin 5 is an important Th2 cytokine involved in controlling the growth, differentiation, and activation of eosinophils, ${ }^{49}$ which densely infiltrate peripheral tissues in $\mathrm{BCL6}^{-/-}$mice. Because production of IL-5 by BCL6 ${ }^{-1-}$ T cells was augmented in vivo and in vitro, we have investigated whether BCL6-related mechanisms regulate IL-5 production, and have identified a BCL6-binding DNA sequence as a silencer (IL5BS) in the 3' untranslated ( $\left.3^{\prime} \mathrm{UT}\right)$ region of the murine and human $I L-5$ genes. $^{15}$

As described above, regulation of IL-4 production by in vitro-differentiated Th2 cells is most likely not regulated by BCL6. However, we have identified putative multiple BCL6binding sites in the IL-4 gene, and BCL6 binds to these sites at rest but not after activation (M. Arima, $\mathrm{MD}, \mathrm{PhD}$, et al, unpublished data, 2008). The physiological roles of BCL6 in lymphocytes with respect to Th2-cell differentiation and the functions of BCL6 such as cytokine production thus remain to be determined.

\section{ROLES OF BCL6 IN CHEMOKINE PRODUCTION}

Toney et $\mathrm{al}^{19}$ have suggested that the development of Th2-type inflammation depends on BCL6 deficiency in some nonlymphoid tissues. They found that three chemokines, monocyte chemoattractant protein 1 (MCP-1), MCP-3, and macrophage inflammatory protein-related protein 1 , were induced at higher levels in BCL6 ${ }^{-1-}$ macrophages than in their BCL6-Wt counterparts. Furthermore, they identify three potential BCL6-binding sites in the promoter of each chemokine. Recently, we found that production of chemokines, including MCP-1, was regulated in airway epithelial cells and macrophages by BCL6. Many CC-type chemokine genes are located in a gene cluster at chromosomes $17 \mathrm{q} 11.2$ and $11 \mathrm{q} 21.2$ in humans and mice, respectively. The cluster includes genes encoding CCL2 (MCP-1) -CCL7 (MCP-3) $C C L 11$ (eotaxin) -CCL8 (MCP-2) -CCL13 (MCP-4) -CCL1 (I-309) in humans and CCL2-CCL7-CCL11-CCL12 (MCP-5) $-C C L 8-C C L 1$ in mice. The strong similarity in the clusters between humans and mice strongly suggests that chemokine expression is orchestrated by the conserved machinery. Because $M C P-1$ (CCL2) is known to be negatively regulated by BCL6 and some putative BCL6-binding sequences are observed in the gene clusters, we examined the role of BCL6 in the expression of these chemokine genes in the cluster. The levels of these chemokine mRNAs other than CCL12 mRNA were strikingly higher in lung tissues and bronchoalveolar lavage fluid cells from BCL6 ${ }^{-1-}$ mice than in those from control mice. Down-regulation of BCL6 in human alveolar epithelial cells (A549) up-regulated the expression of these chemokine genes. Furthermore, chromatin modifications, such as acetylation and methylation of histone, and BCL6 binding to the sequences in the cluster were observed in each cell line on chromatin immunoprecipitation assay (T. Seto, $\mathrm{MD}, \mathrm{PhD}$, et al., unpublished data, 2008). These results suggest that BCL6 promotes Th2 differentiation via other mediators, such as chemokines, in addition to IL-4. This notion is supported by 
evidence that MCP-1 stimulates IL-4 production by $\mathrm{T}$ cells, ${ }^{50}$ $\mathrm{MCP}^{-1} 1^{-/}$mice show very poor Th2 differentiation, ${ }^{51}$ and MCP-1 also suppresses IL-12 secretion from human peripheral blood monocytes. ${ }^{52}$ Thus, BCL6 may play an important role in the coordinated regulation of these chemokine genes in pulmonary epithelial cells and macrophages. BCL6 apparently acts as a transcriptional repressor not only for Th2-cell functions, but also for migration of inflammatory cells, including eosinophils, macrophages, and mast cells. These cells also contribute to Th2-type inflammation and/or Th2-cell differentiation by producing Th2-type cytokines.

\section{FUNCTIONS OF BCL6 IN MACROPHAGES}

BCL6 has been reported to play an important role in the functions of macrophages, as described previously. Furthermore, we have recently identified other effects of BCL6 on IL18 production in macrophages. Interleukin 18 was discovered as a potent interferon- $\gamma$-inducing factor that is produced by macrophages and DCs in response to stimulation with microbes or microbe products. ${ }^{53}$ Recent studies have shown that IL-18 can also promote Th2 cytokine production from T cells, natural killer cells, basophils, and mast cells. ${ }^{54,55}$ Interleukin 18 seems to be involved in human allergic diseases such as atopic dermatitis (AD), bronchial asthma, and rhinitis. Several studies have reported correlations among serum levels of IL-18, IgE, and disease severity in AD and asthma. ${ }^{56-59}$ Furthermore, leukocytes prepared from the peripheral blood of patients with AD produce larger amounts of IL-18 in response to lipopolysaccharide (LPS) than do leukocytes from healthy volunteers. ${ }^{60}$ Interleukin 18 levels in nasal secretions are also higher in patients with allergic rhinitis than in healthy volunteers. $^{61}$

Because IL-18 triggers the differentiation of Th2 cells, we examined the expression of $I L-18$ mRNA in bone marrow-derived macrophages from $\mathrm{BCL}^{-1-}$ mice after LPS stimulation. ${ }^{17}$ The expression of IL-18 was strikingly up-regulated after stimulation. The expression was also upregulated in RAW264 cells, a murine macrophage cell line, by transfection with the dominant negative type of BCL6 gene. We identified a putative BCL6-binding DNA sequence (IL-18BS) upstream of exon 1 of the murine $I L-18$ gene and three IL$18 \mathrm{BSs}$ in the promoter region of the human $I L-18$ gene. Binding of BCL6 in nuclear protein from resting RAW264 cells to murine IL-18BS was detected on gel retardation assay and chromatin immunoprecipitation assay. The binding activity decreased gradually in RAW264 cells after LPS stimulation. However, the amount of BCL6 protein in these cells remained constant over the period examined, suggesting the functional modification of BCL6 protein after stimulation. Furthermore, murine IL-18BS was required for BCL6 to repress the expression of the luciferase reporter gene under control of the IL-18 promoter. Therefore, BCL6 might be a key regulator of IL-18 production by macrophages in allergic diseases.

\section{ROLES OF BCL6 IN MAST CELLS}

Mast cells are distributed throughout vascularized tissues and play a key role in allergic reactions by releasing proinflammatory mediators. Mast cell--derived factors act on immune-competent cells to promote survival of eosinophils, maturation of DCs, and activation of $\mathrm{B}$ and $\mathrm{T}$ cells by producing cytokines such as Th2 cytokines and chemokines such as MCP-1, ${ }^{62-66}$ macrophage inflammatory protein $1 \alpha$, regulated on activation normal T-cell expressed and secreted (RANTES), and eotaxin, which foster Th2 development and eosinophil recruitment. ${ }^{67}$ Available evidence thus suggests that mast cells are an initiator of Th2-type inflammation in $\mathrm{BCL}^{-1-}$ mice. We have therefore investigated the properties and functions of $\mathrm{BCL} 6^{-1-}$ mast cells derived from bone marrow cells cultured with IL-3 (BMMCs). There was no significant difference between BCL6 ${ }^{-1-}$ BMMCs and BCL6Wt BMMCs in the expression of surface FcR1 or c-Kit, in apoptosis induced by deprivation of IL-3, or in degranulation. However, BCL6 ${ }^{-1-}$ BMMCs stimulated by FcRI/IgE crosslinking expressed greater amounts of mRNAs of Th2 cytokines such as $I L-4, I L-5$, and $I L-13$ than BCL6-Wt BMMCs did. Because Th2 cytokines, including IL-4 and IL-13, have an important role in Th2 development, ${ }^{6,69}$ mast cells may be one of the initiators of Th2 dominance in $\mathrm{BCL}^{-/-}$mice. Because the number of intraepithelial mast cells increased in BCL6 ${ }^{-/-}$ mice $^{19}$ and the production of Th2 cytokines was augmented in activated $\mathrm{BCL} 6^{-1-}$ mast cells, BCL6 $6^{-1-}$ mast cells may also be a major effector of Th2-type inflammation in BCL6 $6^{-1-}$ mice. Thus, BCL6 ${ }^{-1-}$ mast cells are one of the initiators of Th2-type inflammation in BCL6 ${ }^{-1-}$ mice.

\section{ROLES OF BCL6 IN ASTHMA}

Interestingly, some BCL6-regulated genes are representative genes implicated in allergic diseases. These genes are critical to the pathogenesis of allergic diseases such as asthma and pollinosis. Recently, we studied the roles of BCL6 in T cells in the development of asthma. To this end, Lck promotercontrolled BCL6 transgenic (Lck-BCL6-Tg) mice and BCL6-Wt mice were sensitized with intraperitoneal injections of ovalbumin. Fourteen days after the antigen challenge, isolated Th2 cells (T1ST2 positive) were stimulated with anti-CD3 antibody. Production of IL-4 by T1ST2 T cells from Lck-BCL6 Tg mice was significantly attenuated as compared with that by BCL6-Wt mice, indicating that Th2 cytokine production was inhibited by BCL6 in T cells (T. Ogasawara, MD, PhD, et al, unpublished data, 2008). We also investigated the effect of T cells overexpressing BCL6 on the development of asthma by transferring splenic CD4 T cells from sensitized mice into nontreated BCL6-Wt B6 mice. Twenty-four hours later, these mice were challenged with ovalbumin. Antigen-induced eosinophilic airway inflammation was observed after transfer of lymphocytes from sensitized BCL6-Wt mice, associated with an elevated IL-4 level in bronchoalveolar lavage fluid after antigen exposure. The airway response and increased IL-4 production were prevented by transfer of lymphocytes from sensitized LckBCL6 Tg mice (T. Ogasawara, et al, unpublished data, 2008). Therefore, BCL6 may prevent the development of asthma by attenuating $\mathrm{Th} 2$ cytokine production in an experimental murine model, and failure of BCL6 repressor activity may be involved in the initiation and/or exacerbation of asthma in humans. 


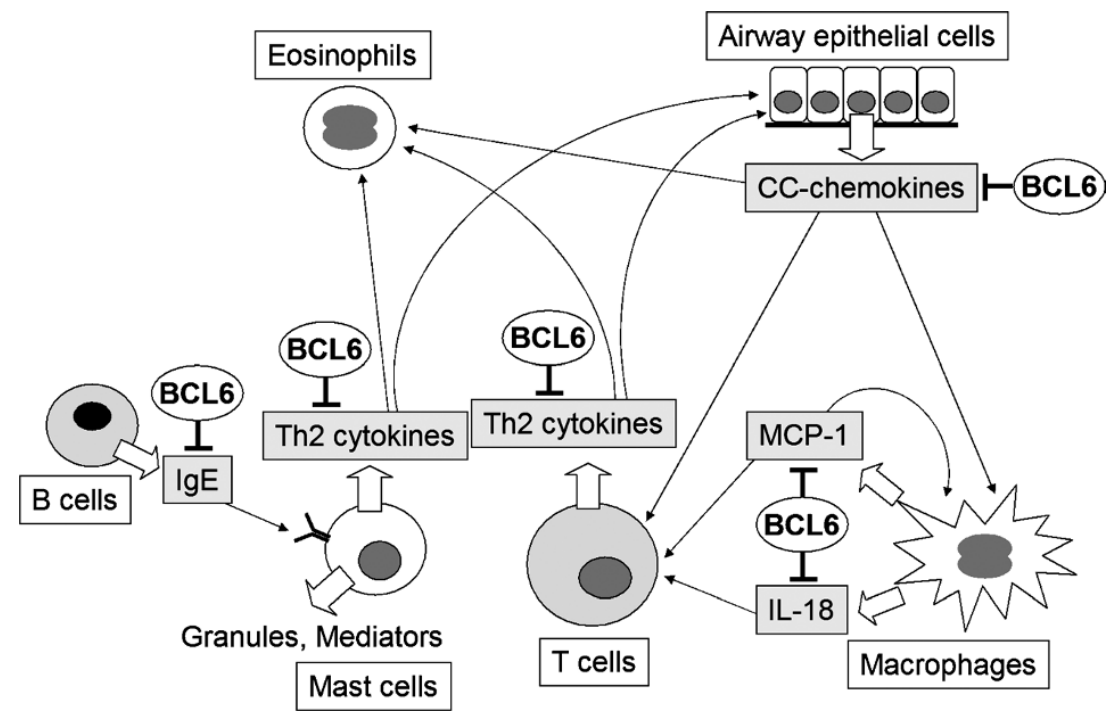

FIGURE 3. Hypothetical role of BCL6 in allergic diseases. BCL6 contributes to the function of a wide range of cells, including T cells, B cells, macrophages, mast cells, and airway epithelial cells to promote the pathogenesis of allergic diseases by regulating genes that control cytokine production, mediator synthesis, and IgE production.

To test the hypothesis that variants of the $B C L 6$ gene might be associated with atopic diseases, Adra et $\mathrm{al}^{70}$ conducted a large-scale association study in both British ( $\mathrm{n}=$ $275)$ and Japanese $(n=400)$ subjects. To identify the exact localization of the known HindIII polymorphism in the first intron of the $B C L 6$ gene $(7 \mathrm{~Kb})$, they first fully sequenced the $7 \mathrm{~Kb}$ clone and determined primer sequences flanking the HindIII polymorphism. They analyzed relations between different types of atopic phenotypes (distinguished by serum $\mathrm{IgE}$ levels) and $B C L 6$ genotypes. Variants of the BCL6 gene were found to be significantly associated with marked atopy, characterized by high IgE levels and positive radioallergosorbent test scores $(>3)$ to both house-dust mite and grass pollen mixtures. However, the functions of the variant of BCL6 remain unclear.

\section{CONCLUSIONS}

In allergic diseases, IL-4-controlled transcriptional regulatory processes might be physiologically regulated by dual molecules, including activators such as STATs and repressors such as BCL6. This control mechanism can be likened to the concept of "yin and yang," involving two primal opposing, but complementary principles. This concept is plausible and critical for preserving biological homeostasis, in which BCL6 may participate in resetting genetic activity. For example, because Th2 cells secrete IL-4 and favor humoral immunity to extracellular pathogens, ${ }^{71}$ BCL6 might attenuate the response. Given that a failure of immune homeostasis leads to the development of allergic diseases, BCL6 may play an important part in such diseases, acting as an inhibitor or protector. Thus, an imbalance between STAT6 and BCL6 may be critically involved in the development of allergic diseases because BCL6 contributes to the function of a wide range of cells, including $\mathrm{T}$ cells, B cells, macrophages, mast cells, and airway epithelial cells (Fig. 3). In these cells, BCL6 contributes to the negative regulation of various key genes that predispose patients to allergies. Therefore, BCL6 may be a major molecular target for Th2-type allergic diseases. However, many important questions remain unresolved, despite considerable progress in understanding gene regulation by BCL6. Further genetic and functional analyses are needed to clarify whether BCL6 is related to the pathogenesis of allergic diseases, with the ultimate goal of establishing more effective means of prophylaxis and therapy. We hope that the studies presented in this review will provide new insights into allergic diseases.

\section{REFERENCES}

1. Dalla-Favera R, Migliazza A, Chang CC, Niu H, Pasqualucci L, Butler M, et al. Molecular pathogenesis of B cell malignancy: the role of BCL-6. Curr Top Microbiol Immunol. 1999;246:257-263.

2. Staudt LM, Dent AL, Shaffer AL, Yu X. Regulation of lymphocyte cell fate decisions and lymphomagenesis by BCL-6. Int J Immunol. 1999; 18:381-403.

3. Dent AL, Shaffer AL, Yu X, Allman D, Staudt LM. Control of inflammation, cytokine expression, and germinal center formation by BCL-6. Science. 1997;276:589-592.

4. Fukuda T, Yoshida T, Okada S, Hatano M, Miki T, Ishibashi K, et al. Disruption of the Bcl6 gene results in an impaired germinal center formation. J Exp Med. 1997;186:439-448.

5. Ye BH, Cattoretti G, Shen Q, Zhang J, Hawe N, de Waard R, et al. The BCL-6 proto-oncogene controls germinal-centre formation and Th2-type inflammation. Nat Genet. 1997;16:161-170.

6. Kerchaert JP, Deweindt C, Tilly H, Quief S, Lecocq G, Bastard C. LAZ3, a novel zinc-finger encoding gene, is disrupted by recurring chromosome 3q27 translocations in human lymphomas. Nat Genet. 1993;5:66-70.

7. Ye BH, Lista F, Lo Coco F, Knowles DM, Offit K, Chaganti RS, et al. Alterations of a zinc finger-encoding gene, $B C L-6$, in diffuse large-cell lymphoma. Science. 1993;262:747-750.

8. Miki T, Kawamata N, Hirosawa S, Aoki N. Gene involved in the 3q27 translocation associated with B-cell lymphoma, $B C L 5$, encodes a Krüppel like zinc-finger protein. Blood. 1994;83:26-32. 
9. Cattoretti G, Chang CC, Cechova K, Zhang J, Ye BH. BCL-6 protein is expressed in germinal-center B cells. Blood. 1995;86:45-53.

10. Yamochi T, Kitabayashi A, Hirokawa M, Miura AB, Onizuka T, Mori S, et al. Regulation of BCL-6 gene expression in human myeloid/ monocytoid leukemic cells. Leukemia. 1997;11:694-700.

11. Baron BW, Stanger RR, Hume E, Sadhu A, Mick R, Kerckaert JP, et al. $B C L 6$ encodes a sequence-specific DNA-binding protein. Genes Chromosomes Cancer. 1995;13:221-224.

12. Seyfert VL, Allman D, He Y, Staudt LM. Transcriptional repression by the proto-oncogene BCL-6. Oncogene. 1996;12:2331-2342.

13. Gupta S, Jiang M, Anthony A, Pernis AB. Lineage-specific modulation of interleukin 4 signaling by interferon regulatory factor 4. J Exp Med. 1999; $190: 1837-1848$

14. Harris MB, Chang CC, Berton MT, Danial NN, Zhang J, Kuehner D, et al. Transcriptional repression of Stat6-dependent interleukin-4-induced genes by BCL-6: specific regulation of iepsilon transcription and immunoglobulin E switching. Mol Cell Biol. 1999;19:7264-7275.

15. Arima M, Toyama H, Ichii $\mathrm{H}$, Kojima S, Okada S, Hatano M, et al. A putative silencer element in the $I L-5$ gene recognized by Bcl6. J Immunol. 2002;169:829-836.

16. Kusam S, Toney LM, Sato H, Dent AL. Inhibition of Th2 differentiation and GATA-3 expression by BCL-6. J Immunol. 2003;170:2435-2441.

17. Takeda N, Arima M, Tsuruoka N, Okada S, Hatano M, Sakamoto A, et al. Bc16 is a transcriptional repressor for the IL-18 gene. J Immunol. 2003;171:426-431.

18. Yu RY, Wang X, Pixley FJ, Yu JJ, Dent AL, Broxmeyer HE, et al. BCL-6 negatively regulates macrophage proliferation by suppressing autocrine IL-6 production. Blood. 2005;105:1777-1784.

19. Toney LM, Cattoretti G, Graf JA, Merghoub T, Pandolfi PP, Dalla-Favera $\mathrm{R}$, et al. BCL-6 regulates chemokine gene transcription in macrophages. Nat Immunol. 2000;1:214-220

20. Shaffer AL, Yu X, He Y, Boldrick J, Chan EP, Staudt LM. BCL-6 represses genes that function in lymphocyte differentiation, inflammation and cell cycle control. Immunity. 2000;13:199-212.

21. Niu H, Cattoretti G, Dalla-Favera R. BCL6 controls the expression of the B7-1/CD80 costimulatory receptor in germinal center B cells. J Exp Med. 2003;198:211-221.

22. Phan RT, Saito M, Basso K, Niu H, Dalla-Favera R. BCL6 interacts with the transcription factor Miz-1 to suppress the cyclin-dependent kinase inhibitor p21 and cell cycle arrest in germinal center B cells. Nat Immunol. 2005;6:1054-1060.

23. Phan RT, Dalla-Favera R. The BCL6 proto-oncogene suppresses p 53 expression in germinal-centre B cells. Nature. 2004;432:635-659.

24. Shvarts A, Brummelkamp TR, Scheeren F, Koh E, Daley GQ, Spits H, et al. A senescence rescue screen identifies BCL6 as an inhibitor of anti-proliferative p19(ARF)-p53 signaling. Genes Dev. 2002;16:681-686.

25. Ranuncolo SM, Polo JM, Dierov J, Singer M, Kuo T, Greally J, et al. Bcl-6 mediates the germinal center B cell phenotype and lymphomagenesis through transcriptional repression of the DNA-damage sensor ATR. Nat Immunol. 2007;8:705-714.

26. Baron BW, Zeleznik-Le N, Baron MJ, Theisler C, Huo D, Krasowski MD, et al. Repression of the PDCD2 gene by BCL6 and the implications for the pathogenesis of human B and T cell lymphomas. Proc Natl Acad Sci U S A. 2007;104:7449-7454.

27. Baron BW, Anastasi J, Thirman MJ, Furukawa Y, Fears S, Kim DC, et al. The human programmed cell death-2 (PDCD2) gene is a target of BCL6 repression: implications for a role of BCL6 in the down-regulation of apoptosis. Proc Natl Acad Sci U S A. 2002;99:2860-2865.

28. Tang TT, Dowbenko D, Jackson A, Toney L, Lewin DA, Dent AL, et al. The forkhead transcription factor AFX activates apoptosis by induction of the BCL-6 transcriptional repressor. J Biol Chem. 2002;277:14255-14265.

29. Li Z, Wang X, Yu RY, Ding BB, Yu JJ, Dai XM, et al. BCL-6 negatively regulates expression of the NF-kappaB1 p105/p50 subunit. $J$ Immunol. 2005; 174:205-214.

30. Harris MB, Mostecki J, Rothman PB. Repression of an interleukin-4responsive promoter requires cooperative BCL-6 function. Biol Chem. 2005;280:13114-13121.

31. Harris MB, Chang CC, Berton MT, Danial NN, Zhang J, Kuehner D, et al. Transcriptional repression of Stat6-dependent interleukin-4-induced genes by BCL-6: specific regulation of iepsilon transcription and immunoglobulin E switching. Mol Cell Biol. 1999;19:7264-7275.
32. Albagli O, Lantoine D, Quief S, Quignon F, Englert C, Kerckaert JP, et al. Overexpressed BCL6 (LAZ3) oncoprotein triggers apoptosis, delays S phase progression and associates with replication foci. Oncogene. 1999;18:5063-5075.

33. Yamochi T, Kaneita Y, Akiyama T, Mori S, Moriyama M. Adenovirus-mediated high expression of BCL-6 in CV-1 cells induces apoptotic cell death accompanied by down-regulation of BCL-2 and BCL-X(L). Oncogene. 1999;18:487-494.

34. Toyama H, Okada S, Hatano M, Takahashi Y. Takeda N, Ichii H, et al. Memory B cells without somatic hypermutation are generated from Bc16-deficient B cells. Immunity. 2002;17:329-339.

35. Ichii H, Sakamoto A, Hatano M, Okada S, Toyama H, Taki S, et al. Role for Bcl-6 in the generation and maintenance of memory CD8+ T cells. Nat Immunol. 2002;3:558-563.

36. Ichii H, Sakamoto A, Kuroda Y, Tokuhisa T. Bcl6 acts as an amplifier for the generation and proliferative capacity of central memory CD8+ T Cells. J Immunol. 2004;173:883-891.

37. Ichii H, Sakamoto A, Arima M, Hatano M, Kuroda Y, Tokuhisa T. Bcl6 is essential for the generation of long-term memory CD4+ T cells. Int Immunol. 2007;19:427-433.

38. Allman D, Jain A, Dent A, Maile RR. Selvaggi T, Kehry MR, et al. BCL-6 expression during B-cell activation. Blood. 1996;87:5257-5268

39. Fero ML, Rivkin M, Tasch M, Porter P, Carow CE, Firpo E, et al. A syndrome of multiorgan hyperplasia with features of gigantism, tumorigenesis, and female sterility in p27(Kip1)-deficient mice. Cell. 1996;85:733-744.

40. Fero ML, Randel E, Gurley KE, Roberts JM, Kemp CJ. The murine gene p27Kip1 is haplo-insufficient for tumour suppression. Nature. 1998;396:177-180.

41. Arguni E, Arima M, Tsuruoka N, Sakamoto A, Hatano M, Tokuhisa T. JunD/AP-1 and STAT3 are the major enhancer molecules for high Bcl6 expression in germinal center B cells. Int Immunol. 2006;18:1079-1089.

42. Kitayama D, Sakamoto A, Arima M, Hatano M, Miyazaki M, Tokuhisa T. A role for Bcl6 in sequential class switch recombination to IgE in B cells stimulated with IL-4 and IL-21. Mol Immunol. 2008;45:1337-1345.

43. Yoshida T, Fukuda T, Hatano M, Koseki H. Okabe S, Ishibashi K, et al. A role of Bcl6 in mature cardiac myocytes. Cardiovasc Res. 1999;42: 670-679.

44. Lund R, Ahlfors H, Kainonen E, Lahesmaa AM, Dixon C, Lahesmaa R. Identification of genes involved in the initiation of human Th1 or Th2 cell commitment. Eur J Immunol. 2005;35:3307-3319.

45. Lund RJ, Ylikoski EK, Aittokallio T, Nevalainen O, Lahesmaa R. Kinetics and STAT4- or STAT6-mediated regulation of genes involved in lymphocyte polarization to Th1 and Th2 cells. Eur J Immunol. 2003;33:1105-1116.

46. Zhou G, Ono SJ. Induction of BCL-6 gene expression by interferon-gamma and identification of an IRE in exon I. Exp Mol Pathol. 2005; 78:25-35

47. Kaplan MH, Schindler U, Smiley ST, Grusby MJ. Stat6 is required for mediating responses to IL-4 and for development of Th2 cells. Immunity. 1996;4:313-319.

48. Dent AL, Hu-Li J, Paul WE, Staudt LM. T helper type 2 inflammatory disease in the absence of interleukin 4 and transcription factor STAT6. Proc Natl Acad Sci U S A. 1998;95:13823-13828.

49. Yamaguchi Y, Hayashi Y, Sugama Y, Miura Y, Kasahara T, Kitamura S, et al. Highly purified murine interleukin 5 (IL-5) stimulates eosinophil function and prolongs in vitro survival. IL-5 as an eosinophil chemotactic factor. $J$ Exp Med. 1988;167:1737-1742.

50. Karpus WJ, Lukacs NW, Kennedy KJ, Smith WS, Hurst SD, Barrett TA. Differential CC chemokine-induced enhancement of T helper cell cytokine production. J Immunol. 1997;158:4129-4136.

51. Gu L, Tseng S, Horner RM, Tam C, Loda M, Rollins BJ. Control of TH2 polarization by the chemokine monocyte chemoattractant protein-1. Nature. 2000;404:407-411.

52. Braun MC, Lahey E, Kelsall BL. Selective suppression of IL-12 production by chemoattractants. $J$ Immunol. 2000;164:3009-3017.

53. Okamura H, Tsutsi H, Komatsu T, Yutsudo M, Hakura A, Tanimoto T, et al. Cloning of a new cytokine that induces IFN-gamma production by T cells. Nature. 1995:378:88-91.

54. Nakanishi K, Yoshimoto T, Tsutsi H, Okamura H. Interleukin-18 is a unique cytokine that stimulates both $\mathrm{Th} 1$ and $\mathrm{Th} 2$ responses depending on its cytokine milieu. Cytokine Growth Factor Rev. 2001;12:53-72. 
55. Sugimoto T, Ishikawa Y, Yoshimoto T, Hayashi N, Fujimoto J, Nakanishi $\mathrm{K}$. Interleukin 18 acts on memory $\mathrm{T}$ helper cells type 1 to induce airway inflammation and hyperresponsiveness in a naive host mouse. $J$ Exp Med. 2004;199:535-545.

56. El-Mezzein RE, Matsumoto T, Nomiyama H, Miike T. Increased secretion of IL-18 in vitro by peripheral blood mononuclear cells of patients with bronchial asthma and atopic dermatitis. J Clin Immunol. 2001;126: 193-198.

57. Yoshizawa Y, Nomaguchi H, Izaki S, Kitamura K. Serum cytokine levels in atopic dermatitis. Clin Exp Dermatol. 2002;27:225-229.

58. Wong CK, Ho CY, Ko FW, Chan CH, Ho AS, Hui DS, et al Proinflammatory cytokines (IL-17, IL-6, IL-18 and IL-12) and Th cytokines (IFN-g, IL-4, IL-10 and IL-13) in patients with allergic asthma. Clin Exp Immunol. 2001;125:177-183.

59. Tanaka H, Miyazaki N, Oashi K, Teramoto S, Shiratori M, Hashimoto M, et al. IL-18 might reflect disease activity in mild and moderate asthma exacerbation. J Allergy Clin Immunol. 2001;107:331-336.

60. Higashi N, Gesser B, Kawana S, Thestrup-Pedersen K. Expression of IL-18 mRNA and secretion of IL-18 are reduced in monocytes from patients with atopic dermatitis. J Allergy Clin Immunol. 2001;108:607-614.

61. Verhaeghe B, Gevaert P, Holtappels G, Lukat KF, Lange B, Van Cauwenberge P, et al. Up-regulation of IL-18 in allergic rhinitis. Allergy. 2002;57:825-830.

62. Levi-Schaffer F, Temkin V, Malamud V, Feld S, Zilberman Y. Mast cells enhance eosinophil survival in vitro: role of TNF-alpha and granulocytemacrophage colony-stimulating factor. J Immunol. 1998;160:5554-5562.

63. Skokos S, Le Panse S, Villa I, Rousselle JC, Peronet R, David B, et al. Mast cell-dependent B and T lymphocyte activation is mediated by the secretion of immunologically active exosomes. J Immunol. 2001;166: 868-876.

64. Skokos D, Botros HG, Demeure C, Morin J, Peronet R, Birkenmeier G, et al. Mast cell-derived exosomes induce phenotypic and functional maturation of dendritic cells and elicit specific immune responses in vivo. J Immunol. 2003;170:3037-3045.

65. Schmitz J, Thiel A, Kuhn R, Rajewsky K, Muller W, Assenmacher M, et al. Induction of interleukin 4 (IL-4) expression in T helper (Th) cells is not dependent on IL-4 from non-Th cells. J Exp Med. 1994;179: 1349-1353.

66. Fallon PG, Jolin HE, Smith P, Emson CL, Townsend MJ, Fallon R, et al IL-4 induces characteristic Th2 responses even in the combined absence of IL-5, IL-9, and IL-13. Immunity. 2002;17:7-17.

67. Nakajima T, Inagaki N, Tanaka H, Tanaka A, Yoshikawa M, Tamari M, et al. Marked increase in CC chemokine gene expression in both human and mouse mast cell transcriptomes following Fcepsilon receptor I cross-linking: an interspecies comparison. Blood. 2002;100:3861-3868.

68. McKenzie GJ, Emson CL, Bell SE, Anderson S, Fallon P, Zurawski G, et al. Impaired development of Th2 cells in IL-13-deficient mice. Immunity. 1998;9:423-432.

69. Chiaramonte G, Donaldson DD, Cheever AW, Wynn TA. An IL-13 inhibitor blocks the development of hepatic fibrosis during a T-helper type 2-dominated inflammatory response. J Clin Invest. 1999;104:777-785.

70. Adra CN, Gao PS, Mao XQ, Baron BW, Pauker S, Miki T, et al. Variants of B cell lymphoma 6 (BCL6) and marked atopy. Clin Genet. 1998;54: 362-364.

71. O'Garra A. Cytokines induce the development of functionally heterogeneous T helper cell subsets. Immunity. 1998;8:275-283. 